\title{
DNA Methylation, Preterm Birth and Blood Pressure in African American Children: The DPREG Study
}

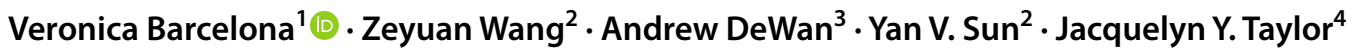

Accepted: 18 April 2021 / Published online: 22 April 2021

(c) The Author(s), under exclusive licence to Springer Science+Business Media, LLC, part of Springer Nature 2021

\begin{abstract}
Preterm birth ( $<37$ weeks) has been associated with high blood pressure (BP) and cardiovascular disease in adulthood. Epigenetic mechanisms may explain how preterm birth influences later BP. In this study, we examined the association between DNA methylation (DNAm), preterm birth and BP in African American children. We recruited 100 children and collected clinical and birth history data. DNA was extracted from saliva and the Illumina EPIC BeadChip was used for epigenetic analyses. Preterm birth was not associated with systolic or diastolic BP. No significant DNAm sites were associated with preterm birth in candidate gene methylation analyses. Body mass index was associated with systolic BP $(\mathrm{p}=0.01)$. We did not observe an effect of preterm birth on DNAm or BP in early childhood. Our study is one of the few, however, to examine these associations among African Americans.
\end{abstract}

Keywords DNA methylation $\cdot$ Preterm birth $\cdot$ Blood pressure $\cdot$ Epigenetics $\cdot$ African Americans $\cdot$ Children

\section{Introduction}

High blood pressure (BP) is the most common risk factor for cardiovascular disease (CVD) in the United States, affecting approximately 116 million adults [1]. African American (AA) adults bear a disproportionately high prevalence of hypertension (40.3\%) compared to Caucasians (27.8\%), Hispanics (27.8\%), and Asians (25.0\%) [2]. In addition, African Americans have an earlier age of hypertension onset and higher rates of sequelae such as end-stage renal disease, stroke, and congestive heart failure than other racial/ethnic groups [3]. Rates of hypertension among children are rising $[4,5]$ and elevated BP in childhood predicts adult

Veronica Barcelona

vb2534@cumc.columbia.edu

1 Columbia University School of Nursing, 560 West 168th St, New York, NY 10032, USA

2 Emory University Rollins School of Public Health, 201 Dowman Drive, 30322 Atlanta, Georgia

3 Center for Perinatal, Pediatric and Environmental Epidemiology, Yale University School of Public Health, 1 Church Street, 6th Floor, New Haven, CT 06510, USA

4 Center for Research on People of Color, Columbia University School of Nursing, 560 W 168th St, New York, NY 10032, USA cardiovascular risk [6]. Approximately $10 \%$ of children aged 8 to 17 had high or borderline high BP in 2011-2012 [5], and rates are increasing among African Americans [7]. More recent prevalence estimates in 5 to 18 year olds using NHANES surveys from 1999 to 2015 reported a rate of 9.3\% [8]. Traditional risk factors for development of early CVD in childhood include dyslipidemia, obesity, diabetes, high $\mathrm{BP}$, family history of premature CVD and cigarette smoke exposure $[9,10]$.

Preterm birth ( $<37$ weeks gestation) is another important risk factor for high BP $[11,12]$. African Americans have the highest rates of preterm birth of any racial/ethnic group in the U.S. (13.6\%, compared to $9.06 \%$ for Caucasians and $9.45 \%$ for Hispanics)[13]. Children born preterm often experience developmental delays and growth stunting leading to an accelerated period of catch-up growth that has been linked with obesity [14] and increased CVD risk [15] in adolescence. A large meta-analysis reported higher mean systolic and diastolic blood pressures $(3.4 \mathrm{mmHg}$ and $2.1 \mathrm{mmHg}$, respectively) in early adulthood for those born preterm compared to those born at term [16]. Though these increases in blood pressure in young adulthood are modest, they can translate to higher stroke risk, for example, in subsequent years $[17,18]$.

Mechanisms for the link between preterm birth and later cardiovascular risk remain unclear. Genomic and 
epigenomic factors have been associated with CVD risk [19, 20], as well as with preterm birth [21-26]. Epigenomics is the study of the complete set of epigenetic modifications on the genetic material of a cell, known as the epigenome.[27] Epigenetic mechanisms produce changes to gene expression and include DNA methylation (DNAm), histone modification and microRNAs. DNAm is the most frequently studied epigenetic mechanism and refers to the addition of a methyl group to the cytosine residues often resulting in suppressing gene expression [28]. Epigenetic mechanisms produce changes to gene expression which are heritable and reversible, yet do not involve alterations to the DNA sequence. Epigenetic mechanisms such as DNAm have most frequently been studied in relation to cancer and chronic disease endpoints such as autoimmune disorders, mental illnesses, and diabetes [29-32].

There is a paucity of studies on DNA methylation (DNAm) effects in children born preterm, especially among African Americans. Parets et al. [21] examined umbilical blood at delivery in a cohort of African American women who delivered preterm (24-34 weeks; $\mathrm{n}=22)$ and at term (39-41 weeks, $n=28$ ). They reported 29 DNAm sites associated with preterm birth independent of gestational age. A small case-control study reported associations between DNAm alteration in placenta and cord blood and newborns born preterm compared to those born full-term [22]. Another study identified differential methylation patterns in whole blood in early childhood by gestational age in European ancestry samples [23].

Small case-control studies of Asian samples also contribute to the limited literature in this area. One included 32 spontaneous preterm births and 16 term births from South China [22]. They reported one differentially methylated position in placenta and 31 positions in cord blood that were associated with preterm birth but noted differences in target tissues. Others examined preterm $(n=68)$ and term $(n=951)$ infants in an Asian cohort and associations in cord tissue and cord blood with genome-wide methylation [24]. They found that preterm birth was significantly associated with DNAm at 296 DNAm sites in cord blood, and over 95\% of these were replicated in other preterm birth epigenome-wide association study (EWAS) cohorts, and that novel sites were identified in cord tissue that were unique from those identified in cord blood.

In an all-Caucasian Australian sample extreme preterm birth cases $(n=12)$ and term controls $(n=12)$ from dried blood spots at birth were compared to DNA from blood at 18 years of age.[25] They reported 1,555 sites with significant methylation differences between term and preterm babies, but that these differences had largely resolved by 18 years. The authors suggest that DNAm differences at birth may be due to cell composition and gestational age, however 10 genomic loci were identified that persisted into late adolescence. Finally, the Avon Longitudinal Study of Parents and Children[26] examined Cord blood DNA at birth $(n=914)$ and peripheral blood at $7(n=973)$ and 17 $(n=974)$ years of age to compare changes at differentially methylated probes in relation to birthweight and gestational age. The authors reported methylation at 224 DNAm sites associated with gestational age, and 23 DNAm sites associated with birthweight at follow up ages, with resolution of differential methylation by early childhood.

Therefore, the purpose of this study was to examine the association between preterm birth, DNAm, and high BP among African American children. Given the available sample size and because we were only interested in targeting genes associated with preterm birth, we used a candidate gene approach instead of EWAS. We hypothesized that children with increased methylation of candidate genes associated with preterm birth would have higher BP than those born at term.

\section{Theoretical Framework}

This study was informed by Bronfenbrenner's bioecological theory [33], which states that child development results from an interaction between the individual and their environment. The elements of this theory include: (1) the person's biology and genomics, (2) reciprocal interactions between the child and the immediate environment, (3) direct or indirect environmental influences; and (4) time.

\section{Methods}

\section{Participants}

The DNA methylation, Preterm birth, and BP in African American Children (DPREG) study was conducted between November 2017 and May 2020. DPREG was a K01 funded sub-study of the Intergenerational Impact of Genetic and Psychological Factors on Blood Pressure Study (InterGEN) [34]. Briefly, InterGEN enrolled 250 African American mother/child dyads $(\mathrm{N}=500)$ from 2014 to 2019 for a longitudinal study. Dyads contributed clinical, genetic, and psychological data at four visits, six months apart (Time 1-Time 4). At the Time 1 InterGEN visit, salivary DNA was collected from mothers and their biological child (aged 3-5 years), as well as height, weight, and BP. Demographic and psychological variables were at each visit. Mothers' eligibility for participation in InterGEN included that they: (1) spoke English, (2) were $\geq 21$ years old, (3) self-identified as African American or Black, and 4) had no psychological impairment.

The DPREG study enrolled 100 children from the InterGEN cohort. Children were targeted for enrollment 
if mothers identified in their birth history at the Time 2 InterGEN visit that the child was born preterm. Eligibility requirements for enrollment in DPREG included that the child was: (1) a past/current participant in InterGEN, (2) a singleton gestation, and (3) willing to participate per the child's mother. DPREG study aims included examination of how preterm birth was associated with DNAm and BP in enrolled children. Medical records were abstracted for pregnancy and birth history. Mothers were compensated (\$15) for DPREG participation. Written, informed consent was obtained, and all study procedures were approved by the affiliated institutional review board. The datasets analyzed during the current study are available from the corresponding author on reasonable request.

\section{Data Collection and Measures}

Trained research assistants conducted field data collection according to established protocols. BP was measured manually, three times according to pediatric guidelines [35], with the seated child at rest for five minutes before measurement with the appropriately sized cuff. Height was measured to the nearest tenth of an inch on barefoot participants and weight was measured using a high-capacity electronic scale (Tanita Tokyo, Japan). We collected self-reported pregnancy history and reviewed medical records for pregnancy and birth outcomes. Smoking (during pregnancy and current) was ascertained at Time 1 .

InterGEN quality assurance protocols were followed for salivary DNA collection, extraction and analysis as previously described [36]. Participants were asked not to eat, drink, smoke, or chew gum for $30 \mathrm{~min}$ before passive saliva was collected in Oragene-500 Format tubes [37]. Saliva collection tubes were barcode labeled to ensure precise sample tracking and logged into a computerized freezer inventory once received in the laboratory. Saliva samples were refrigerated at $4{ }^{\circ} \mathrm{C}$ until DNA extraction and analysis were completed using standard operating procedures and Reliaprep kits. The Illumina Infinium Methylation EPIC (850 K) BeadChip was used to ascertain DNA methylation (DNAm) for candidate gene analyses [38]. Detailed epityping and analysis procedures have been previously described [36]. Quantile-normalization of beta values for autosomal DNAm sites was performed. All individual samples passed laboratory-based quality-control procedures (missing rate $<10 \%$ and no sex mismatch). DNAm sites were excluded if they had a missing rate greater than $10 \%$, overlapped with SNPs, or were listed in the recent Illumina product quality notice. Candidate DNAm sites and gene regions from previous reports were identified by unique DNAm identifiers or chromosomal positions from reference human genome (Supplementary Tables S1 and S2).

\section{Statistical Analysis}

We conducted a cross-sectional analysis of DNAm, preterm birth and blood pressure in DPREG children at the Time 1 InterGEN visit. The main outcome (dependent) variable was continuous BP (continuous variable, mean of three readings). We examined systolic and diastolic BPs separately in models. The exposure (independent) variables were DNAm (continuous beta values) and preterm birth and gestational age. Preterm birth was treated as a dichotomous variable ( $<37$ weeks, and $\geq 37$ weeks), and gestational age at birth was examined as a continuous variable. Similarly, low birthweight was examined as a continuous and dichotomous variable $(<2500 \mathrm{~g}$, and $\geq 2500 \mathrm{~g})$. Confounders were identified a priori; including age, sex, obesity and exposure to current maternal smoking. Child BMI $\left(\mathrm{kg} / \mathrm{m}^{2}\right)$ was determined by a percentile scale based on sex and age according to 2000 CDC Growth Charts ( 0 to $<20$ years). (CDC 2019) BMI was categorized as underweight $(<5$ th percentile), normal weight $\left(5^{\text {th }}\right.$ to $84^{\text {th }}$ percentile), overweight (85th to 94 th percentile), and obesity (at or above the $95^{\text {th }}$ percentile) [39, 40], and also examined as a continuous variable. Descriptive statistics and bivariate analyses were conducted using frequencies and Pearson Correlation Coefficients, and linear and logistic regression models were employed to examine relationships between gestational age, birthweight and BPs. Phenotype analyses were conducted using SAS 9.4 (Cary, N.C.).

To examine the extent to which methylation in candidate genes mediated the association between preterm birth and BP, we used a two-step approach. We first examined the associations between candidate genes/DNAm sites previously identified in the literature and preterm birth in our sample. Then, any significant associations would be tested in the full model with DNAm, preterm birth, and BP as the outcome. Linear mixed effects models were used to model the dependent variable (DNAm) for systolic and diastolic BP separately, adjusting for age, sex, maternal smoking, and cell type heterogeneity. We adjusted for multiple comparisons by applying False Discovery Rate for correction of the alpha. Primary analyses were conducted to account for these accepted confounders in epigenetic studies, as well as batch effects. The reference-free EWAS method was used to account for potential heterogeneity in cell proportions from saliva samples [41]. Epigenomic analyses were done using R statistical analysis software.

\section{Results}

A total of 99 children contributed data for the present study. One child was excluded for multiple gestation. Objective birth history from medical records was obtained for $\mathrm{n}=77$ 
participants, due to a halt in research operations related to the COVID-19 pandemic. Maternal self-report was used for participants missing abstraction data for birth, as gestational age and birthweight were highly correlated $(\mathrm{p}<0.0001$ for both birthweight and gestational age) in this sample.

Characteristics of participating children and their mothers at the Time 1 visit are presented in Table 1 . The mean age of children enrolled in DPREG was 49.9 months (4.1 years), and more were female (58.5\%) than male (41.4\%). Approximately two thirds of mothers of enrolled children had achieved some college or higher education, and most (86.3\%) reported an annual household income of $\$ 50,000$ or less. Mothers were insured most commonly through Medicaid $(59.6 \%)$. Over a quarter of mothers $(26.2 \%)$ reported a history of high BP and about a quarter $(24.1 \%)$ reported being current smokers. Mean child BMI was 16.1, in the normal range, $11.1 \%$ of children were overweight and $10.1 \%$ were obese. The mean gestational age of enrolled children was 38 weeks, with $16.3 \%$ born preterm ( $<37$ weeks). Mean birthweight was $3073 \mathrm{~g}$, and $10.3 \%$ were low birthweight $(<2500 \mathrm{~g})$.

We then examined bivariate associations between selected child and maternal demographics and mean systolic and diastolic BP (data not shown). Child sex, child age and maternal smoking were not significantly associated with either systolic or diastolic BPs. Continuous BMI was significantly associated with systolic BP (Pearson Correlation Coefficient $=0.25$, $\mathrm{p}$-value $=0.01)$ but not diastolic $\mathrm{BP}$ (Pearson Correlation Coefficient $=0.13$, $\mathrm{p}$-value $=0.19$ ). Linear regression models (Table 2) revealed no association between gestational age and preterm birth with systolic or diastolic BP before and after adjustment for child age, maternal smoking, and child BMI. Birthweight was also not significantly associated with systolic or diastolic BPs.Linear mixed effects models were used to examine DNAm of candidate gene sites identified previously, adjusting for child age, sex, principal components, and cell type heterogeneity. The experimental batches and methylation chips were modeled as random effects in the linear mixed effect models to adjust for potential batch effects on epigenetic association analysis. We examined 24 previously reported DNAm sites associated with preterm birth, and none were found significant in our data (Supplementary Table S1). The DNAm sites from eight previously identified genes associated with preterm birth are summarized and annotated in Supplementary Table S2.

We also conducted sensitivity analyses and found that those enrolled in DPREG $(n=100)$ did not differ from those approached but not enrolled $(n=71)$ on maternal demographic variables or smoking at Time 1 (data not shown). The groups did differ on systolic BP at baseline $(p=0.01)$, as enrolled children had lower systolic BP (mean $=89.05$, $\mathrm{SD}=8.8 \mathrm{mmHg}$ ) than those who were not enrolled (mean $=92.35, \mathrm{SD}=7.9 \mathrm{mmHg}$ ). There were no statistically
Table 1 Maternal and child characteristics of participants enrolled in the DNA methylation, preterm birth and blood pressure in African American children study (DPREG), 2017-2020, $n=99$

\begin{tabular}{|c|c|c|}
\hline Maternal characteristics & $\mathrm{n}(\text { mean })^{\mathrm{a}}$ & 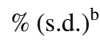 \\
\hline \multicolumn{3}{|l|}{ Age } \\
\hline $20-29$ & 38 & 38.3 \\
\hline $30-39$ & 55 & 55.5 \\
\hline $40-49$ & 6 & 6.0 \\
\hline \multicolumn{3}{|l|}{ Highest education completed } \\
\hline$<$ High School & 3 & 3.0 \\
\hline High School graduate & 32 & 32.3 \\
\hline Some college & 30 & 30.3 \\
\hline Associate degree/College graduate or higher & 34 & 34.3 \\
\hline \multicolumn{3}{|l|}{ Annual household income } \\
\hline$\leq \$ 15,000$ & 40 & 41.6 \\
\hline$>\$ 15,000-\$ 50,000$ & 43 & 44.7 \\
\hline$\geq \$ 50,000$ & 13 & 13.5 \\
\hline \multicolumn{3}{|l|}{ Health insurance } \\
\hline Private/employer & 18 & 18.1 \\
\hline Medicaid & 59 & 59.6 \\
\hline Government/ACA & 16 & 16.1 \\
\hline Other & 4 & 4 \\
\hline Uninsured & 2 & 2 \\
\hline \multicolumn{3}{|l|}{ History of high blood pressure } \\
\hline Yes & 26 & 26.2 \\
\hline No & 73 & 73.7 \\
\hline \multicolumn{3}{|l|}{ Current smoker } \\
\hline Yes & 24 & 24.4 \\
\hline No & 74 & 75.5 \\
\hline \multicolumn{3}{|l|}{ Child characteristics } \\
\hline Age (months) & 49.9 & 8.6 \\
\hline $\mathrm{BMI}^{\mathrm{c}}\left(\mathrm{kg} / \mathrm{m}^{2}\right)$ & 16.1 & 1.6 \\
\hline \multicolumn{3}{|l|}{ BMI-for-age } \\
\hline Underweight (<5th percentile) & 2 & 2.0 \\
\hline Normal weight (5th to $<85$ percentile) & 76 & 76.7 \\
\hline Overweight (85th to <95th percentile) & 11 & 11.1 \\
\hline Obese ( $\geq 95$ th percentile) & 10 & 10.1 \\
\hline Systolic blood pressure (mmHg) & 89.0 & 8.9 \\
\hline Diastolic blood pressure (mmHg) & 58.4 & 7.2 \\
\hline \multicolumn{3}{|l|}{ Sex } \\
\hline Male & 41 & 41.4 \\
\hline Female & 58 & 58.5 \\
\hline Gestational age at birth (weeks) & 38.0 & 2.9 \\
\hline \multicolumn{3}{|l|}{ Preterm } \\
\hline Yes ( $\leq 37$ weeks gestation) & 16 & 16.3 \\
\hline No (term birth > 37 weeks) & 82 & 83.6 \\
\hline Birthweight (grams) & 3073.0 & 688.4 \\
\hline \multicolumn{3}{|l|}{ Low birthweight } \\
\hline Yes $(<2500 \mathrm{~g})$ & 10 & 10.3 \\
\hline No $(\geq 2500 \mathrm{~g})$ & 87 & 89.6 \\
\hline
\end{tabular}

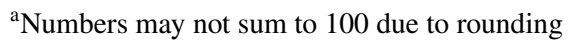

${ }^{\mathrm{b}}$ s.d. $=$ standard deviation

${ }^{\mathrm{c}}$ Body Mass Index

$k g$ kilogram, $m$ meters 


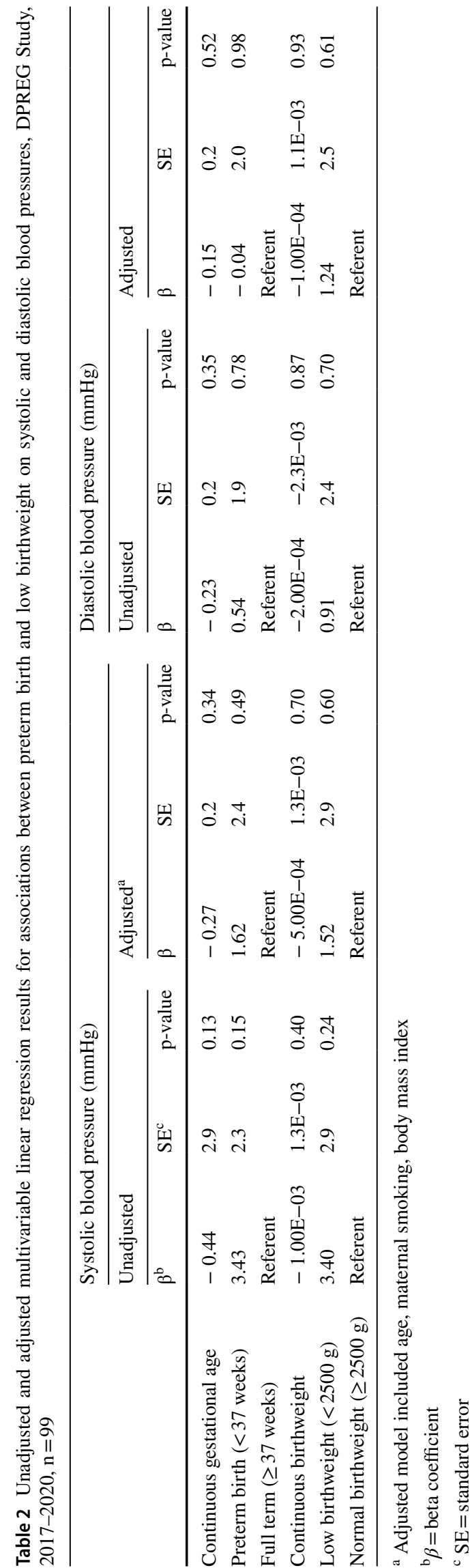

significant differences $(\mathrm{p}>0.05)$ between enrolled and unenrolled children at Time 1 for diastolic BP $(p=0.43)$, child BMI $(p=0.20)$, or gestational age (maternal self-report at Time 2) $(\mathrm{p}=0.99)$.

\section{Discussion}

In this study examining the relationship between preterm birth, DNAm and BP in African American children, child BMI was associated with systolic BP $(p=0.01)$ in bivariate analyses, though preterm birth status, gestational age and birthweight were not associated with systolic or diastolic $\mathrm{BP}$ in either adjusted or unadjusted analyses. We found no epigenetic associations with preterm birth in candidate gene analysis. Our findings differed from previous epigenomic studies of preterm birth and DNAm. We used findings from Parets et al. [21] for comparison in our analyses. Our findings differed from that study, and this may be explained by more restrictive categorization of full-term birth (defined as 24-34 weeks gestation), and their use of cord blood for DNAm analyses, perhaps indicating differences in methylation by target tissue.

We did observe that higher BMI in children was significantly associated with systolic BP in bivariate analyses. This unadjusted association supports well-established findings that obesity is a significant and prevalent clinical risk factor for high BP in children [42]. In the United States in 2015-2016, the prevalence of obesity among children aged $2-5$ years was $13.9 \%$ and between 6 and 11 years was $18.4 \%$. [43] Among African American children aged 2-5 years, $21.9 \%$ were overweight or obese according to the 2011-2012 NHANES survey.[44] Approximately $28 \%$ of children in our sample were overweight or obese, who ranged in age from 4 to 9 years (mean $=6$ years).

In addition, we found that preterm birth status and birthweight were not significantly associated with BP in the young children in our study. Though several large studies have examined this relationship, our study is unique in that most existing studies have not included children this young, nor have they been conducted in an all-African American sample [45]. Our findings are in line with one large study of Caucasians and African Americans which reported no increased risk of high BP at 7 years of age in children born small for gestational age [46].

Our findings may have differed from other studies for several reasons. Our target tissue was saliva, while previous studies used umbilical cord blood, peripheral blood, or placenta. Saliva is a relevant tissue as it contains DNA from both epithelial cells and leukocytes and is non-invasive. Leukocytes isolated from saliva reflect a biological immune system marker which is a key indicator of BP. The lack of studies on the association between preterm birth 
and DNAm in saliva limit comparability of studies and underscore the need for more research on various tissue types and among African Americans. Our previous work identified novel sites associated with cigarette smoking and DNAm in saliva in the same InterGEN cohort, which were replicated in blood samples of African American women in the GENOA cohort [47]. One explanation for this may be that the signal related to smoking was stronger in mothers than the effect of preterm birth on children in early childhood.

In addition, our sample of preterm births was smaller in this study than anticipated. This limited the power available to detect associations where they were present. In addition, differential misclassification may have occurred in mothers' reporting of preterm birth for those children who were missing medical record data. However, previous research has found that maternal self-report of gestational age is a valid measure, even years after birth [48, 49]. Due to the limited number of DPREG children who were born preterm, we were unable to more precisely parse out DNAm differences related to spontaneous and elective preterm birth. Only 7 of the 16 children born preterm in this sample had confirmed preterm labor and spontaneous preterm birth documented in available medical records.

Our study findings may not be generalizable as this was a community-based convenience sample, and we targeted children born preterm for enrollment. Children enrolled in DPREG had lower BPs than those who were not enrolled. This could have biased our results in the direction of the null. The use of pediatric blood pressure in research is known to be vulnerable to measurement error, though a recent study revealed acceptable agreement between manual and automatic measurement methods [50]. We minimized the potential for measurement error in this study by using a small group of trained research assistants who collected clinical data from participants in both the larger parent study as well as the current DPREG study.

In conclusion, our study adds to the sparse literature on the association of preterm birth, DNAm and childhood $\mathrm{BP}$ in African Americans. Though we did not find significant associations, our study is one of the few to examine this question in this vulnerable population. Considering the significant burden of preterm birth and CVD among African Americans, future studies are warranted to explore additional epigenomic markers and biological mechanisms associated with preterm birth and later cardiovascular health. A prospective approach may clarify epigenetic patterns and high BP onset in African American children and have implications for prevention.

Supplementary Information The online version contains supplementary material available at https://doi.org/10.1007/s10903-021-01201-y.
Acknowledgements This work was supported by the National Institute for Nursing Research [K01NR017010, R01NR013520].

Author Contributions Each of the following authors (VB, JYT, YVS, $\mathrm{AW})$ contributed to the conceptualization of this study and the revision of the manuscript. Veronica VB, JYT, YVS, AW, and ZW reviewed and edited the manuscript. VB conducted data collection and drafted the original manuscript. VB, YVS and ZW conducted statistical analyses.

\section{Declarations}

Conflicts of interest The authors declare that they have no conflict of interest.

Ethics Approval This study was performed in line with the principles of the Declaration of Helsinki. Approval was granted by the Institutional Review Board of Yale University (HIC\#2000020775).

Informed Consent Informed consent was obtained from study participants (mothers) and assent given for their children's participation.

\section{References}

1. Virani SS, Alonso A, Benjamin EJ, Bittencourt MS, Callaway CW, Carson AP, et al. Heart disease and stroke statistics-2020 update: a report from the American Heart Association. Circulation. 2020 Jan 29;141(9):e139-596.

2. Fryar CD, Ostchega Y, Hales CM, Zhang G, Kruszon-Moran D. Hypertension prevalence and control among adults: United States, 2015-2016. NCHS Data Brief. 2017 Oct;289:1-8.

3. Carnethon MR, Pu J, Howard G, Albert MA, Anderson CAM, Bertoni AG, et al. Cardiovascular health in African Americans: a scientific statement From the American Heart Association. Circulation. 2017 Nov 21;136(21):e393-423.

4. McNiece KL, Poffenbarger TS, Turner JL, Franco KD, Sorof JM, Portman RJ. Prevalence of hypertension and pre-hypertension among adolescents. J Pediatr. 2007;150(6):640-4.

5. Kit BK, Kuklina E, Carroll MD, Ostchega Y, Freedman DS, Ogden CL. Prevalence of and trends in dyslipidemia and blood pressure among US children and adolescents, 1999-2012. JAMA Pediatr. 2015 Mar;169(3):272-9.

6. Theodore RF, Broadbent J, Nagin D, Ambler A, Hogan S, Ramrakha $\mathrm{S}$, et al. Childhood to early-midlife systolic blood pressure trajectories: early-life predictors, effect modifiers, and adult cardiovascular outcomes. Hypertension. 2015 Dec;66(6):1108-15.

7. Din-Dzietham R, Liu Y, Bielo MV, Shamsa F. High blood pressure trends in children and adolescents in national surveys, 1963 to 2002. Circulation. 2007 Sep 25;116(13):1488-96.

8. Sharma AK, Metzger DL, Rodd CJ. Prevalence and severity of high blood pressure among children based on the 2017 American Academy of Pediatrics Guidelines. JAMA Pediatr. 2018 Jun 1;172(6):557-65.

9. de Ferranti SD, Steinberger J, Ameduri R, Baker A, Gooding H, Kelly AS, et al. Cardiovascular risk reduction in high-risk pediatric patients: a scientific statement from the American Heart Association. Circulation. 2019;139(13):e603-34.

10. Expert Panel on Integrated Guidelines for Cardiovascular Health and Risk Reduction in Children and Adolescents, National Heart, Lung, and Blood Institute. Expert panel on integrated guidelines for cardiovascular health and risk reduction in children and adolescents: summary report. Pediatrics. 2011;128(5):S213-56. 
11. de Jong F, Monuteaux MC, van Elburg RM, Gillman MW, Belfort MB. Systematic review and meta-analysis of preterm birth and later systolic blood pressure. Hypertension. 2012;59(2):226-34.

12. Bensley JG, De Matteo R, Harding R, Black MJ. The effects of preterm birth and its antecedents on the cardiovascular system. Acta Obstet Gynecol Scand. 2016; 95(6):652-63.

13. Martin JA, Osterman MJK. Describing the increase in preterm births in the United States, 2014-2016. NCHS Data Brief. 2018;312(312):1-8.

14. Ou-Yang MC, Sun Y, Liebowitz M, Chen CC, Fang ML, Dai W, et al. Accelerated weight gain, prematurity, and the risk of childhood obesity: a meta-analysis and systematic review. PLoS ONE. 2020;15(5):e0232238.

15. Inomata $S$, Yoshida $T$, Koura U, Tamura K, Hatasaki K, Imamura $\mathrm{H}$, et al. Effect of preterm birth on growth and cardiovascular disease risk at school age. Pediatr Int. 2015;57(6):1126-30.

16. Hovi P, Vohr B, Ment LR, Doyle LW, McGarvey L, Morrison $\mathrm{KM}$, et al. Blood Pressure in young adults born at very low birth weight: adults born preterm international collaboration. Hypertension. 2016;68(4):880-7.

17. Chen X, Wang Y. Tracking of blood pressure from childhood to adulthood: a systematic review and meta-regression analysis. Circulation. 2008 Jun 24;117(25):3171-80.

18. Lewington S, Clarke R, Qizilbash N, Peto R, Collins R. Prospective Studies Collaboration: Age-specific relevance of usual blood pressure to vascular mortality: a meta-analysis of individual data for one million adults in 61 prospective studies. Lancet. 2002;360(9349):1903-13.

19. Arnett DK, Claas SA. Omics of blood pressure and hypertension. Circ Res. 2018 May 11;122(10):1409-19.

20. Westerman K, Sebastiani P, Jacques P, Liu S, DeMeo D, Ordovás JM. DNA methylation modules associate with incident cardiovascular disease and cumulative risk factor exposure. Clin Epigenetics. 2019;1(1):142.

21. Parets SE, Conneely KN, Kilaru V, Fortunato SJ, Syed TA, Saade $\mathrm{G}$, et al. Fetal DNA methylation associates with early spontaneous preterm birth and gestational age. PLoS ONE. 2013 Jun 27;8(6):e67489.

22. Wang XM, Tian FY, Fan LJ, Xie CB, Niu ZZ, Chen WQ. Comparison of DNA methylation profiles associated with spontaneous preterm birth in placenta and cord blood. BMC Med Genomics. 2019;12(1):1018.

23. Merid SK, Novoloaca A, Sharp GC, Küpers LK, Kho AT, Roy $\mathrm{R}$, et al. Epigenome-wide meta-analysis of blood DNA methylation in newborns and children identifies numerous loci related to gestational age. Genome Med. 2020;12(1):25.

24. Wu Y, Lin X, Lim IY, Chen L, Teh AL, MacIsaac JL, et al. Analysis of two birth tissues provides new insights into the epigenetic landscape of neonates born preterm. Clin Epigenetics. 2019;11(1):26.

25. Cruickshank MN, Oshlack A, Theda C, Davis PG, Martino D, Sheehan P, et al. Analysis of epigenetic changes in survivors of preterm birth reveals the effect of gestational age and evidence for a long term legacy. Genome Med. 2013 Oct 18;5(10):96.

26. Simpkin AJ, Suderman M, Gaunt TR, Lyttleton O, McArdle WL, Ring SM, et al. Longitudinal analysis of DNA methylation associated with birth weight and gestational age. Hum Mol Genet. 2015 Jul 1;24(13):3752-63.

27. Dupont C, Armant DR, Brenner CA. Epigenetics: definition, mechanisms and clinical perspective. Semin Reprod Med. 2009 Sep;27(5):351-7.

28. Moore LD, Le T, Fan G. DNA methylation and its basic function. Neuropsychopharmacology. 2013 Jan;38(1):23-38.

29. Klutstein M, Nejman D, Greenfield R, Cedar H. DNA methylation in cancer and aging. Cancer Res. 2016 Jun 15;76(12):3446-50.
30. Ehrlich M. DNA hypermethylation in disease: mechanisms and clinical relevance. Epigenetics. 2019 Dec;14(12):1141-63.

31. Bansal A, Pinney SE. DNA methylation and its role in the pathogenesis of diabetes. Pediatr Diabetes. 2017 May;18(3):167-77.

32. Stevens AJ, Rucklidge JJ, Kennedy MA. Epigenetics, nutrition and mental health: Is there a relationship? Nutr Neurosci. 2018;21(9):602-13.

33. Bronfenbrenner U. Article 1. The Bioecological Theory of Human Development. Making human beings human: Bioecological perspectives on human development Thousand Oaks, CA: Sage; 2005. pp. 3-15.

34. Crusto CA, de Mendoza BV, Connell CM, Sun YV, Taylor JY. The Intergenerational Impact of Genetic and Psychological Factors on Blood Pressure Study (InterGEN): Design and Methods for Recruitment and Psychological Measures. Nurs Res. 2016;65(4):331-8.

35. National High Blood Pressure Education Program Working Group on High Blood Pressure in Children and Adolescents: The fourth report on the diagnosis, evaluation, and treatment of high blood pressure in children and adolescents. Pediatrics 2004; 114(2):555-76.

36. Taylor JY, Wright ML, Crusto CA, Sun YV. The intergenerational impact of genetic and psychological factors on blood pressure (InterGEN) study: design and methods for complex DNA analysis. Biol Res Nurs. 2016.

37. Bahlo M, Stankovich J, Danoy P, Hickey PF, Taylor BV, Browning SR, et al. Saliva-derived DNA performs well in large-scale, high-density single-nucleotide polymorphism microarray studies. Cancer Epidemiol Biomark Prev. 2010;19(3):794-8.

38. Illumina. Infinium ${ }^{\circledR}$ Expanded Multi-Ethnic Genotyping Array (MEGAEX); A Consortium-Built Array with Increased Power for Understanding Complex Disease in Diverse Human Populations. Illumina Inc. 2015.

39. Krebs NF, Himes JH, Jacobson D, Nicklas TA, Guilday P, Styne D. Assessment of child and adolescent overweight and obesity. Pediatrics. 2007 Dec;120(4):S193-228.

40. Kuczmarski RJ, Ogden CL, Guo SS, Grummer-Strawn LM, Flegal KM, Mei Z, et al. 2000 CDC growth charts for the United States: methods and development. Vital Health Stat. 2002;246(246):1-190.

41. Houseman EA, Molitor J, Marsit CJ. Reference-free cell mixture adjustments in analysis of DNA methylation data. Bioinformatics. 2014 May 15;30(10):1431-9.

42. Huang RC, Prescott SL, Godfrey KM, Davis EA. Assessment of cardiometabolic risk in children in population studies: underpinning developmental origins of health and disease mother-offspring cohort studies. J Nutr Sci. 2015 Apr;10(4):e12.

43. Hales CM, Carroll MD, Fryar CD, Ogden CL. Prevalence of obesity among adults and youth: United States, 2015-2016. NCHS Data Brief. 2017;288(288):1-8.

44. Ogden CL, Carroll MD, Kit BK, Flegal KM. Prevalence of childhood and adult obesity in the United States, 2011-2012. JAMA. 2014 Feb 26;311(8):806-14.

45. Edvardsson VO, Steinthorsdottir SD, Eliasdottir SB, Indridason OS, Palsson R. Birth weight and childhood blood pressure. Curr Hypertens Rep. 2012 Dec;14(6):596-602.

46. Hemachandra AH, Howards PP, Furth SL, Klebanoff MA. Birth weight, postnatal growth, and risk for high blood pressure at 7 years of age: results from the Collaborative Perinatal Project. Pediatrics. 2007 Jun;119(6):e1264-70.

47. Barcelona V, Huang Y, Brown K, Liu J, Zhao W, Yu M, et al. Novel DNA methylation sites associated with cigarette smoking among African Americans. Epigenetics. 2019 Apr;14(4):383-91.

48. Petersen JM, Mitchell AA, Van Bennekom C, Werler MM. Validity of maternal recall of gestational age and weight at birth: 
comparison of structured interview and medical records. Pharmacoepidemiol Drug Saf. 2019 Feb;28(2):269-73.

49. Carter EB, Stuart JJ, Farland LV, Rich-Edwards JW, Zera CA, McElrath TF, et al. Pregnancy complications as markers for subsequent maternal cardiovascular disease: validation of a maternal recall questionnaire. J Womens Health (Larchmt). 2015 Sep;24(9):702-12.

50. Varney EJ, Van Drunen AM, Moore EF, Carlin K, Thomas K. Blood Pressure measurement error in children: lessons in measurement reliability. J Nurs Meas. 2019 Apr 1;27(1):114-25.
Publisher's Note Springer Nature remains neutral with regard to jurisdictional claims in published maps and institutional affiliations. 